\title{
Tunable Electronic Structure and Magnetic Coupling in Strained Two-Dimensional Semiconductor $\mathrm{MnPSe}_{3}$
}

\author{
Qi Pei ${ }^{1}$, Xiaocha Wang ${ }^{2}$, Jijun Zou ${ }^{3}$, Wenbo $\mathrm{Mi}^{1{ }^{1, *}}$ \\ ${ }^{1}$ Tianjin Key Laboratory of Low Dimensional Materials Physics and Preparation Technology, \\ School of Science, Tianjin University, Tianjin 300354, China
}

${ }^{2}$ School of Electrical and Electronic Engineering, Tianjin University of Technology, Tianjin 300384, China

${ }^{3}$ Key Laboratory for Green Chemical Technology of the Ministry of Education, School of Chemical Engineering and Technology, Tianjin University, Tianjin 300354, China

\footnotetext{
*Author to whom all correspondence should be addressed.

E-mail: $\underline{\text { miwenbo@tju.edu.cn }}$
} 


\begin{abstract}
The electronic structures and magnetic properties of strained monolayer $\mathrm{MnPSe}_{3}$ are investigated systematically by first-principles calculations. It is found that the magnetic ground state (GS) of monolayer $\mathrm{MnPSe}_{3}$ can be significantly affected by biaxial strain engineering, while the semiconducting characteristics are well preserved. Owing to the sensitivity of the magnetic coupling towards the structural deformation, a biaxial tensile strain about $13 \%$ can lead to an antiferromagnetic-ferromagnetic (AFM-FM) transition. The underlying physical mechanism of strain-dependent magnetic stability is mainly attributed to the competition effect of direct AFM interaction and indirect FM superexchange interaction between the nearest-neighbor (NN) two Mn atoms. In addition, we find that $\mathrm{FM} \mathrm{MnPSe}$ is an intrinsic half semiconductor with a large spin exchange splitting in conduction bands, which is crucial for the spin-polarized carrier injection and detection. The sensitive interdependence among external stimuli, electronic structure and magnetic coupling suggests that monolayer $\mathrm{MnPSe}_{3}$ can be a promising candidate in spintronics.
\end{abstract}

Keywords: two-dimensional semiconductor, $\mathrm{MnPSe}_{3}$, strain engineering, electronic structure, magnetic coupling

PACS: 71.15.Mb, 73.22.-f, 75.50.Pp, 75.30.Et 


\section{Introduction}

Recently, the emergence of two-dimensional (2D) materials has aroused widespread concerns [1-4]. Especially, 2D magnetic semiconductors who possess both semiconducting and magnetic characteristics have been considered as promising candidates for low-dimensional spintronic devices. In general, magnetic semiconductors can be classified into two categories: one is intrinsic magnetic semiconductor and the other is diluted magnetic semiconductor. Compared with intrinsic magnetic semiconductor, the diluted magnetic semiconductor can be easily obtained by doping the nonmagnetic semiconductor with transition metals [5-7]. However, there still exist some problems in synthesis, such as low solubility and rich boundary defects. Therefore, searching for the experimentally feasible methods for the fabrication of magnetic semiconductors is full of importance. At present, the strategy of exfoliating single layers from bulk layered structures has been comprehensively investigated. Following this approach, recent theoretical studies have proposed some potential magnetic 2D van der Waals (vdW) materials, including transition metal carbides and nitride $M X$ enes [8], chromium-based ternary tritellurides $\mathrm{Cr} X \mathrm{Te}_{3}(X=\mathrm{Si}, \mathrm{Ge})$ [9-11], vanadium-based dichalcogenides $\mathrm{V}_{2}(X=\mathrm{S}, \mathrm{Se})$ [12], trihalides $\operatorname{Cr} X_{3}(X=\mathrm{F}, \mathrm{Cl}, \mathrm{Br}, \mathrm{I})$ [13] and transition metal phosphorus trichalcogenides $\mathrm{MPX}_{3}(M=\mathrm{Fe}, \mathrm{Mn}, \mathrm{Ni} ; X=\mathrm{S}, \mathrm{Se})$ [14-16]. As one of the representative in $M P X_{3}$ family, monolayer $\mathrm{MnPSe}_{3}$ exhibits particular advantages in solar-energy-related applications and novel spin-valley coupling physics [17, 18], which endows this $2 \mathrm{D}$ crystal with great potentials in spintronics. 
The control of the spin ordering is another key issue for the application of spintronic devices.

The spin ordering of magnetic materials should be deliberately modulated by external stimuli, such as electric field, atomic vacancy, adsorption, doping, or elastic strain engineering [19-22]. Among these external factors, mechanical strain is commonly regarded as an effective route to induce the change of crystal structure, the modification of orbital overlap and the emergence of charge distribution. Meantime, the excellent mechanical flexibility of 2D magnets provides further proof of the strain-engineering's feasibility [23, 24]. Many theoretical reports have shown the tunable electronic structures and magnetic characteristics in stained monolayers [12, 25-28]. Experimentally, a remarkable advance in applying tunable biaxial strain to 2D materials has been proposed by Ding et al. [29]. Using a thin layer of polymethyl methacrylate (PMMA) as glue, single layer graphene can be transferred onto a $\mathrm{SiO}_{2}$ covered piezoelectric substrate. Then a bias voltage applied to the piezoelectric substrate will result in an out-of-plane electric field and subsequently lead to an in-plane strain. This technique not only makes it possible to study strain-related behaviors of low-dimensional materials but also inspires new ideas and methods in strain application.

In this paper, the electronic and magnetic properties of monolayer $\mathrm{MnPSe}_{3}$ are investigated by density functional theory systemically, as well as the intercoupling between the strain and magnetism. It is found that the pristine monolayer $\mathrm{MnPSe}_{3}$ is an intrinsic semiconductor with antiferromagnetic (AFM) ordering. The stability of AFM coupling can be significantly weakened by applying the elastic biaxial strains. An antiferromagnetic-ferromagnetic (AFM-FM) transition occurs under a large tensile strain beyond $13 \%$. The physical mechanism of such a phenomenon is further studied, and a strain-related competition between direct exchange interaction and indirect 
superexchange interaction is put forward. In addition, monolayer $\mathrm{MnPSe}_{3}$ with $\mathrm{FM}$ coupling exhibits robust semiconducting characteristics, with fully spin-polarized valance and conduction band edges. These novel properties render monolayer $\mathrm{MnPSe}_{3}$ a promising platform to explore magnetic phenomena and offer a promising avenue for fabricating controllable and tunable spintronic devices.

\section{Computational details}

Our calculations are performed in the framework of density functional theory (DFT), using a plane-wave basis [30] set as implemented in the VASP code [31]. The generalized gradient approximation (GGA) parameterized by Perdew-Burke-Ernzerhof (PBE) [32] is utilized to describe exchange-correlation functional. For the reason that GGA cannot properly describe the strongly correlated systems with partially filled $d$ subshells, we use Hubbard $U$ terms (5 eV for Mn) to describe the on-site electron-electron Coulomb repulsion as suggested in the literature [33]. The vdW-D2 correction [34] is used together with GGA+U to add the longer-ranged correlation in evaluating vdW interaction between the monolayers. Kohn-Sham single-particle wavefunctions are expanded in the plane wave basis set with a kinetic energy truncation at $500 \mathrm{eV}$. The Monkhorst-Pack scheme is adopted for $k$-point sampling employing a $7 \times 7 \times 1 \Gamma$-centered grid. The energy and force convergence criteria on each atom are less than $10^{-6} \mathrm{eV}$ and $0.01 \mathrm{eV} / \AA$, respectively. A $2 \times 2 \times 1$ supercell model including eight $\mathrm{Mn}$ atoms is adopted in calculation to indentify the preferred magnetic ground state (GS), and a $20 \AA$ vacuum slab is inserted 
perpendicularly on the top of $\mathrm{MnPSe}_{3}$ surface to minimize the interaction between periodic images.

\section{Results and discussion}

Bulk $\mathrm{MnPSe}_{3}$ has been proposed to be an AFM semiconductor with the optical energy gap of $2.27 \mathrm{eV}$ [35] and Neél temperature of $74 \mathrm{~K}$ [36]. Since the relatively weak vdW force holds together the layered compound, monolayer $\mathrm{MnPSe}_{3}$ is easily exploited from its corresponding bulk phase. As shown in Figs. 1(a) and (b), each $\mathrm{MnPSe}_{3}$ unit cell is composited of two $\mathrm{Mn}^{2+}$ ions and one $\left[\mathrm{P}_{2} \mathrm{Se}_{6}\right]^{4-}$ cluster. A Mn atom locates at the center of a distorted octahedron with six Se atoms arranged in a trigonal antiprism structure. The dumbbell-like $\mathrm{P}$ dimer in $\left[\mathrm{P}_{2} \mathrm{Se}_{6}\right]^{4-}$ bipyramid constrains six neighboring Se atoms to form two Se trimers with a relative in-plane twist of $60^{\circ}$, perpendicular to the honeycomb plane. The formal valance of $\mathrm{Mn}, \mathrm{P}$ and Se atoms are $+2,+4$ and -2 , respectively. According to Hund's rule, $\mathrm{Mn}^{2+}$ with a $3 d^{5}$ electronic configuration will exhibit a high spin state.

Lattice structure of $\mathrm{MnPSe}_{3}$ monolayer is optimized to observe the resulting lattice parameters. After fully relaxation, the lattice constant of the FM structure $(6.409 \AA)$ is quite similar to the AFM (6.403 $\AA$ ), suggesting the irrelevance between the lattice parameter and magnetic structure. The agreement of optimized lattice constant with the experimental value (6.387 $\AA)$ [36] also reveals that $\mathrm{GGA}+U$ can correctly describe the structural and electronic properties of monolayer $\mathrm{MnPSe}_{3}$, verifying the rationality of our parameters in calculation. The spin density for FM and AFM configurations are calculated by the equation of $\rho=\rho_{\uparrow}-\rho_{\downarrow}$, where the $\rho_{\uparrow}$ and $\rho_{\downarrow}$ represent 
spin-up and spin-down charge densities, respectively. As illustrated in Fig. 1(c), the major magnetism in monolayer $\mathrm{MnPSe}_{3}$ with FM ordering is contributed by Mn atoms. The local magnetic moment of $\mathrm{Mn}$ atom is textured to be about $4.613 \mu_{\mathrm{B}}$, and each $\mathrm{P}$ and Se atom carries about 0.016 and $0.023 \mu_{\mathrm{B}}$ magnetic moments. Similarly, the spin density for AFM configuration in Fig. 1(d) also concentrates on two $\mathrm{Mn}$ atoms with opposite spin magnetic moment $\left(4.598 \mu_{\mathrm{B}}\right)$, while the magnetic moment of each $\mathrm{P}$ and Se can be neglected. The total magnetic moment of AFM form is exactly $0 \mu_{\mathrm{B}}$ for the intrinsic AFM coupling of $\mathrm{Mn}^{2+}$ ions.

To confirm the most preferable magnetic coupling between the Mn atoms, with the exception of FM and regular AFM (Néel) orders, we consider two additional AFM configurations, which are zigzag-AFM and stripy-AFM shown in Fig. 1(e). After differentiate them energetically, the relative energy differences $\Delta E$ (absolute value) with respect to the Néel-AFM configuration are $0,37.77$, 13.12 and $17.92 \mathrm{meV}$ for Néel-AFM, FM, zigzag-AFM and stripy-AFM, respectively. Our calculations clearly show that the Néel-AFM order has the minimum energy, indicating the GS of Néel order. Unless indicated otherwise, AFM order will refer to the AFM-Néel order in the following. Besides, the non-magnetic state can be neglected for the great energy disparity between the NM state and magnetic states.

To evaluate the effect of strain engineering on the electronic and magnetic properties, a series of $x y$-plane biaxial strains are applied to monolayer $\mathrm{MnPSe}_{3}$. As shown in Fig. 2(c), we stretch or shrunk the lattice constants uniformly to simulate the tensile and compressive strains. The strain can be estimated by considering the lattice constants quantitatively as $\varepsilon=\left(a-a_{0}\right) / a_{0}$, where $a_{0}$ and $a$ are the lattice constants of the original and strained models, respectively. A distinction between 
tensile and compressive strains is made via defining $\varepsilon>0$ or $\varepsilon<0$. The variation of energy difference between the AFM and FM coupling in biaxial strained systems is depicted in Fig. 2(a). With the tensile strain changes from $0 \%$ to $5 \%$, the energy difference $\Delta E_{\mathrm{AFM}-\mathrm{FM}}$ increases from -37.77 to $-20.30 \mathrm{meV}$ per formula unit, suggesting that the AFM state is significantly weakened and tends towards instability. When the tensile strain continues to increase, a clear magnetic phase transition from AFM to FM is achieved in the presence of a relatively large strain about $13 \%$. Moreover, the FM stability can be further enhanced under a $15 \%$ tensile strain. Since large strain modulations (beyond 10\%) have already been reported in many ultrathin 2D systems, such as graphene [37] and $\mathrm{MoS}_{2}$ [25] by using either the three-point bending configuration or piezoelectric substrates, we are quite right in believing that $13 \%$ tensile strain can also be realized in monolayer $\mathrm{MnPSe}_{3}$. Hence, strain engineering can be considered as a potential modulating artifice to realize the transition of magnetic phase in monolayer $\mathrm{MnPSe}_{3}$. Meanwhile, the distance between the nearest-neighbor (NN) two $\mathrm{Mn}$ atoms increases linearly from 3.515 to $4.267 \AA$ as the biaxial strain varying from -5\% to $15 \%$. Actually, we calculate the total energies for all possible magnetic configurations under each biaxial strain, and the variation of energy difference $\left(\Delta E_{\mathrm{AFM} \text {-zigzag }}\right.$ and $\left.\Delta E_{\mathrm{AFM} \text {-stripy }}\right)$ is shown in Fig. $\mathrm{S} 1$ in the Supplementary Information. Since there is no other magnetic phase transition occurring within the biaxial strain range we consider, we only focus on the AFM to FM transition.

Fig. 2(b) shows the variation of band gap in biaxial strained systems. Within the strain region from $-5 \%$ to $3 \%$, the band gap increases gradually. However, when the tensile strain increases continuously, band gap values begin to decrease. A sharp band gap shrinking from 1.09 to $0.67 \mathrm{eV}$ appears at the tensile strain of $13 \%$, corresponding to the magnetic phase transition from AFM to 
FM. In addition, the robustness of semiconducting property against wide range of biaxial strain intensity also should be noted.

Band structure (BS) and density of states (DOS) within the energy window ranging from -3 to $3 \mathrm{eV}$ are calculated to reveal the electronic properties in strained monolayer MnPSe 3 . Figs. 3(a)-(f) denote to the systems under $-5 \%, 0 \%, 5 \%, 10 \%, 13 \%$ and $15 \%$ strains, respectively. For the pristine monolayer (see Fig. 3(b)), $\mathrm{MnPSe}_{3}$ is a semiconductor with a direct band gap of $1.83 \mathrm{eV}$, in accordance with previous theoretical results $[14,17]$. The valence bands (VB) near Fermi level mainly consist of Se and Mn atom states, whereas the conduction bands (CB) are contributed by Se, Mn, and P atoms. A comparison of Figs. 3(c) and (e) shows that, as the lattice constants increase, the contribution of the Se $p$ orbitals to the states in the range of VBM to $-0.5 \mathrm{eV}$ increases. The peak of the Se PDOS in this energy range shifts to higher energies. Moreover, by applying a 5\% tensile strain, BS calculations reveal an indirect semiconductor with the conduction band minimum and valance band maximum at $\Gamma$ and $K$ points, respectively. The band transition from direct to indirect also makes the gap decrease to $1.64 \mathrm{eV}$, which is well consistent with the variation tendency given in Fig. 2(b). With the strain increased to 13\%, FM state is more stable than AFM state. The occupied states near Fermi level have major contribution from Se atoms, while the unoccupied states in the vicinity of Fermi level are deriving from Se, Mn, and P atoms. Examination of the PDOS of Mn and Se atoms shows that $\mathrm{Mn} d$ states are relatively delocalized and hybridized with Se $p$ states, indicating that indirect $p-d$ exchange interaction plays an important role in the ferromagnetic coupling. In addition, $\mathrm{FM} \mathrm{MnPSe}{ }_{3}$ is an indirect semiconductor with the band gap of $0.66 \mathrm{eV}$. The bands in the spin-up and spin-down channels are not overlapped, suggesting the intrinsic 
ferromagnetism. Particularly, the VB and $\mathrm{CB}$ edges of $\mathrm{FM} \mathrm{MnPSe}_{3}$ are fully spin-polarized in the same spin direction, indicating that the $\mathrm{FM} \mathrm{MnPSe}$ is a half semiconductor (HSC). A large spin exchange splitting of $0.40 \mathrm{eV}$ (labled as $\Delta$ in Fig. 3(e)) in the $\mathrm{CB}$ is observed, which is not only essential for the spin-polarized carrier injection and detection [10], but also crucial for gaining half-metallic property by shifting the relative position of Fermi level. The exchange splitting can be further increased to $0.44 \mathrm{eV}$ at a tensile strain of $15 \%$.

Because our calculations show that the magnetic moments are concentrated on the metal atoms sites (see Fig. 1(c)-(d)), we characterize the magnetic properties of $\mathrm{MnPSe}_{3}$ with an effective Heisenberg model Hamiltonian on a honeycomb lattice,

$$
H=\sum_{<i j>} J_{1} \mathbf{S}_{i} \cdot \mathbf{S}_{j}+\sum_{<i j>} J_{2} \mathbf{S}_{i} \cdot \mathbf{S}_{j}+\sum_{<i j>} J_{3} \mathbf{S}_{i} \cdot \mathbf{S}_{j}
$$

where $J_{1,2,3}$ are the exchange interactions between $\mathrm{NN}$, second $\mathrm{NN}$, and third $\mathrm{NN}$ spins. $\mathbf{S}_{i}$ is the total spin magnetic moment of the atomic site $i$. To further extract the magnetic exchange interactions, the lattice is fixed to that of the most energetically favorable spin confirmation and the energies for different spin configurations are computed. Using equation (1), the magnetic energy can be explicitly expressed as

$$
\begin{aligned}
& E_{\mathrm{FM} / \mathrm{N} \text { él }}=E_{0}+\left( \pm 3 J_{1}+6 J_{2} \pm 3 J_{3}\right)|\mathbf{S}|^{2} \\
& E_{\text {zigzag/stripy }}=E_{0}+\left( \pm J_{1}-2 J_{2} \mathrm{~m} 3 J_{3}\right)|\mathbf{S}|^{2}
\end{aligned}
$$

We then obtain the lattice constant, GS along with the exchange coupling constants for pristine and biaxial strained $\mathrm{MnPSe}_{3}$. As listed in Table S1, the $\mathrm{NN}$ interaction $J_{1}$, the second $\mathrm{NN}$ interaction $J_{2}$ and the third NN interaction $J_{3}$ are all AFM. The value of $J_{2}(0.02 \mathrm{meV})$ is one magnitude less 
than $J_{1}(0.195 \mathrm{meV})$ and much smaller than $J_{3}(0.103 \mathrm{meV})$. These findings are consistent with previous studies on $\mathrm{MnPS}_{3}$ and $\mathrm{MnPSe}_{3}[16,38,39]$. More importantly, $J_{2}$ and $J_{3}$ interactions in transition-metal trichalcogenide monolayers are always AFM [40].

To understand the microscopic origin of the exchange interactions in monolayer $\mathrm{MnPSe}_{3}$, the possible electron hopping paths for $J_{1}, J_{2}$ and $J_{3}$ interactions are plotted. As shown in Fig. 4(a), electrons hopping for $J_{1}$ interaction mainly go through two paths. One is the short-range direct interaction between two neighboring $\mathrm{Mn}$ ions (Mn-Mn), which is robustly AFM for the half-filled high-spin $d^{5}$ state of $\mathrm{Mn}$, and the other is the more long-range Mn-Se-Mn superexchange interaction with an angle of $84.1^{\circ}$. According to the well-known Goodenough-Kanamori-Anderson (GKA) rules $[41,42]$, systems with cation-anion-cation bond angles of $90^{\circ}$ prefer weak FM ordering and $180^{\circ}$ superexchange interactions are AFM. Therefore, the Mn-Se-Mn angle $\left(84.1^{\circ}\right)$ close to $90^{\circ}$ in superexchange is FM. Due to the closed $d$ shell on Mn ions and large electron excitation energy from Se $p$ orbital to $\mathrm{Mn} d$ orbital, the direct AFM exchange interaction dominates over FM superexchange interaction. As a consequence, $J_{1}$ is expected to be AFM.

In Figs. 4(b) and (c), there are several hopping paths for $J_{2}$ and $J_{3}$, where the cations are separated by two anions. Based on the geometry, the most possible paths should involve two se anions on the same plane with the shortest distance. For this reason, $J_{2}$ and $J_{3}$ can be regarded as super-superexchange interactions. Since the rules for cation-anion-anion-cation path are similar to those for cation-anion-cation interactions, $180^{\circ}$ and $90^{\circ}$ are also two criterion angles for AFM and FM interactions, respectively. However, for those interactions with intermediate angels between $90^{\circ}$ and $180^{\circ}$, a crossover angle of $127 \pm 0.6^{\circ}$ is related to the AFM-FM transition [43]. Zhang et al. [44] 
also proposed that a large cation-anion-anion angle beyond $130^{\circ}$ leads to AFM interaction, while an angel about $90^{\circ}$ contributes an FM interaction. According to the above mentioned, we find that the most possible electron hopping path for $J_{3}$ contains two cation-anion-anion angels, which are both $132.3^{\circ}$, resulting in strongly AFM contributions. As for $J_{2}$ interaction, although two cation-anion-anion angles of $132.1^{\circ}$ and $89.5^{\circ}$ contribute to AFM and FM respectively, the resulting extended $J_{2}$ still exhibits AFM sign due to the AFM dominance in monolayer $\mathrm{MnPSe}_{3}$ [44]. Besides, compared with $J_{1}$ and $J_{3}, J_{2}$ is relatively weak for involving small Se-Se hybridizations. Hence, we suppose that the AFM-FM transition may mainly depend on the significant competition effect between AFM exchange interaction and FM superexchange interaction from the NN interaction $J_{1}$. With the increase of biaxial strain, the cation-anion-anion angels always change within the criterion angle range. Values of $J_{2}$ and $J_{3}$ are expected to reduce in magnitude because of the increasing atomic distances, while the variation of $J_{1}$ is subtler. A rapid reduction of the direct exchange interaction and a relatively slow reduction of the indirect superexchange interaction will give rise to the impairment of AFM stability and resultant enhancement of FM stability. Once the tensile strain is large enough, the magnetic phase reversal can be realized by changing the signs (positive and negative) of the energy difference between AFM and FM, and thus the AFM-FM transition can be well explained.

Additionally, the uniaxial strain effect on magnetic phase transition has been recognized in previous $\mathrm{CrPS}_{4}$ monolayer [45]. In this work, uniaxial strain engineering is further tested. The results are listed in Table S2 in the Supplementary Information. Generally, both the uniform biaxial and uniaxial strains could trigger the modification in total energies and result in the magnetic phase 
transition. However, the effect is weaker for uniaxial strain in monolayer $\mathrm{MnPSe}_{3}$. A relatively large uniaxial strain is required to alter the AFM-FM transition.

\section{Conclusion}

In summary, the electronic structures and magnetic properties of the strained monolayer $\mathrm{MnPSe}_{3}$ are systematically surveyed. Our ab initio calculations show that the $2 \mathrm{D} \mathrm{MnPSe}_{3}$ is an AFM semiconductor. In particular, the AFM stability can be substantially influenced by strain engineering and an AFM to FM transition occurs under a large tensile strain, indicating that the strain can be used as an effective knob for modulating the magnetic phase. The underlying physical mechanism of strain-dependent magnetic stability is further elucidated as the competition effect of direct exchange and indirect superexchange interactions between the $\mathrm{NN}$ two $\mathrm{Mn}$ atoms. The research discussed here provides strong evidence that strain can be an effective pathway to induce or modulate magnetic properties in 2D crystal and sheds light on the potential candidate of $\mathrm{MnPSe}_{3}$ as a promising $2 \mathrm{D}$ magnet. 


\section{Acknowledgements}

This work is supported by National Natural Science Foundation of China (51671142, U1632152 and 51661145026) and Key Project of Natural Science Foundation of Tianjin City (16JCZDJC37300). 


\section{REFERENCES}

1. K. S. Novoselov, A. K. Geim, S. V. Morozov, D. Jiang, Y. Zhang, S. V. Dubonos, I. V. Grigorieva, A. A. Firsov, Electric field effect in atomically thin carbon films, Science 306(5696), $666(2004)$

2. K. S. Novoselov, A. K. Geim, S. V. Morozov, D. Jiang, M. I. Katsnelson, I. V. Grigorieva, S. V. Dubonos, A. A. Firsov, Two-dimensional gas of massless Dirac fermions in graphene, Nature 438(7065), 197 (2005)

3. Y. Zhang, Y. W. Tan, H. L. Stormer, P. Kim, Experimental observation of the quantum Hall effect and Berry's phase in graphene, Nature 438(7065), 201 (2005)

4. A. K. Geim, K. S. Novoselov, The rise of graphene, Nat. Mater. 6(3), 183 (2007)

5. F. Banhart, J. Kotakoski, A. V. Krasheninnikov, Structural defects in graphene, ACS Nano 5(1), $26(2010)$

6. H. Pan, J. B. Yi, L. Shen, R. Q. Wu, J. H. Yang, J. Y. Lin, Y. P. Feng, J. Ding, L. H. Van, J. H. Yin, Room-temperature ferromagnetism in carbon-doped ZnO, Phys. Rev. Lett. 99(12), 127201 (2007)

7. C. Cao, M. Wu, J. Jiang, H. P. Cheng, Transition metal adatom and dimer adsorbed on graphene: Induced magnetization and electronic structures, Phys. Rev. B 81(20), 205424 (2010)

8. M. Naguib, V. N. Mochalin, M. W. Barsoum, Y. Gogotsi, 25th anniversary article: MXenes: a new family of two-dimensional materials, Adv. Mater. 26(7), 992 (2014) 
9. S. Lebegue, T. Björkman, M. Klintenberg, R. M. Nieminen, O. Eriksson, Two-dimensional materials from data filtering and ab initio calculations, Phys. Rev. X 3(3), 031002 (2013)

10. X. Li, J. Yang, $\mathrm{Cr} X \mathrm{Te}_{3}(X=\mathrm{Si}, \mathrm{Ge})$ nanosheets: two dimensional intrinsic ferromagnetic semiconductors, J. Mater. Chem. C 2(34), 7071 (2014)

11. M. W. Lin, H. L. Zhuang, J. Yan, T. Z. Ward, A. A. Puretzky, C. M. Rouleau, Z. Gai, L. Liang, V. Meunier, B. G. Sumpter, P. Ganesh, P. R. C. Kent, D. B. Geohegan, D. G. Mandrus, K. Xiao, Ultrathin nanosheets of $\mathrm{CrSiTe}_{3}$ : a semiconducting two-dimensional ferromagnetic material, $J$. Mater. Chem. C 4(2), 315 (2016)

12. Y. Ma, Y. Dai, M. Guo, C. Niu, Y. Zhu, B. Huang, Evidence of the existence of magnetism in pristine $\mathrm{V} X_{2}$ monolayers $(X=\mathrm{S}, \mathrm{Se})$ and their strain-induced tunable magnetic properties, $A C S$ Nano 6(2), 1695 (2012)

13. W. B. Zhang, Q. Qu, P. Zhu, C.H. Lam, Robust intrinsic ferromagnetism and half semiconductivity in stable two-dimensional single-layer chromium trihalides, J. Mater. Chem. C 3(48), 12457 (2015)

14. X. X. Li, X. J. Wu, J. L. Yang, Half-metallicity in $\mathrm{MnPSe}_{3}$ exfoliated nanosheet with carrier doping, J. Am. Chem. Soc. 136(31), 11065 (2014)

15. X. Zhang, X. Zhao, D. Wu, Y. Jing, Z .Zhou, $\mathrm{MnPSe}_{3}$ monolayer: a promising 2D visible-light photohydrolytic catalyst with high carrier mobility, Adv. Sci. 3(10), 1600062 (2016)

16. B. L. Chittari, Y. Park, D. Lee, M. Han, A. H. MacDonald, E. Hwang, J. Jung, Electronic and magnetic properties of single-layer $M P X_{3}$ metal phosphorous trichalcogenides, Phys. Rev. $B$ 94(18), $184428(2016)$ 
17. X. Li, T. Cao, Q. Niu, J. Shi, J. Feng, Coupling the valley degree of freedom to antiferromagnetic order, Proc. Natl. Acad. Sci. U.S.A. 110(10), 3738 (2013)

18. Q. Pei, Y. Song, X. Wang, J. Zou, W. Mi, Superior electronic structure in two-dimensional $\mathrm{MnPS}_{\mathrm{e} 3} / \mathrm{MoS}_{2}$ van der Waals heterostructures, Sci. Rep. 7(1), 9504 (2017)

19. K. F. Mak, C. H. Lui, J. Shan, T. F. Heinz, Observation of an electric-field-induced band gap in bilayer graphene by infrared spectroscopy, Phys. Rev. Lett. 102(25), 256405 (2009)

20. Y. Zhou, Z. Wang, P. Yang, X. Zu, L. Yang, X. Sun, F. Gao, Tensile strain switched ferromagnetism in layered $\mathrm{NbS}_{2}$ and $\mathrm{NbSe}_{2}$, ACS Nano 6(11), 9727 (2012)

21. Y. C. Cheng, Q. Y. Zhang, U. Schwingenschlögl, Valley polarization in magnetically doped single-layer transition-metal dichalcogenides, Phys. Rev. B 89(15), 155429 (2014)

22. K. Sawada, F. Ishii, M. Saito, S. Okada, T. Kawai, Phase control of graphene nanoribbon by carrier doping: appearance of noncollinear magnetism, Nano Lett. 9(1), 269 (2009)

23. F. Li, Z. Chen, Tuning electronic and magnetic properties of $\mathrm{MoO}_{3}$ sheets by cutting, hydrogenation, and external strain: a computational investigation, Nanoscale 5(12), 5321 (2013)

24. H. H. Pérez-Garza, E.W. Kievit, G.F. Schneider, U. Staufer, Highly strained graphene samples of varying thickness and comparison of their behavior, Nanotechnology 25(46), 465708 (2014)

25. S. Bertolazzi, J. Brivio, A. Kis, Stretching and breaking of ultrathin $\mathrm{MoS}_{2}$, ACS Nano 5(12), $9703(2011)$

26. X. Chen, J. Qi, D. Shi, Strain-engineering of magnetic coupling in two-dimensional magnetic semiconductor $\mathrm{CrSiTe}_{3}$ : competition of direct exchange interaction and superexchange 
interaction, Phys. Lett. A 379(1-2), 60 (2015)

27. Y. Ma, Y. Dai, M. Guo, C. Niu, L. Yu, B. Huang, Strain-induced magnetic transitions in half-fluorinated single layers of BN, GaN and graphene, Nanoscale 3(5), 2301 (2011)

28. L. Kou, C. Tang, W. Guo, C. Chen, Tunable magnetism in strained graphene with topological line defect, ACS Nano 5(2), 1012 (2011)

29. F. Ding, H. Ji, Y. Chen, A. Herklotz, K. Dörr, Mei, Y., A. Rastelli, O. G. Schmidt, Stretchable graphene: a close look at fundamental parameters through biaxial straining, Nano Lett. 10(9), $3453(2010)$

30. G. Kresse, J. Furthmüller, Efficient iterative schemes for ab initio total-energy calculations using a plane-wave basis set, Phys. Rev. B 54(16), 11169 (1996)

31. G Kresse, J Furthmüller, Efficiency of ab-initio total energy calculations for metals and semiconductors using a plane-wave basis set, Comput. Mater. Sci. 6 (1), 15 (1996).

32. J. P. Perdew, K. Burke, M. Ernzerhof, Generalized gradient approximation made simple, Phys. Rev. Lett. 77(18), 3865 (1996)

33. S. L. Dudarev, G. A. Botton, S. Y. Savrasov, C. J. Humphreys, A. P. Sutton, Electron-energy-loss spectra and the structural stability of nickel oxide: an LSDA+U study, Phys. Rev. B 57(3), 1505 (1998)

34. S. Grimme, Semiempirical GGA-type density functional constructed with a long-range dispersion correction, J. Comp. Chem. 27(15), 1787 (2006)

35. V. Grasso, L. Silipigni, Optical absorption and reflectivity study of the layered $\mathrm{MnPSe}_{3}$ seleniophosphate, J. Opt. Soc. Am. B 16(1), 132 (1999) 
36. A. Wiedenmann, J. R. Mignod, A. Louisy, R. Brec, J. Rouxel, Neutron diffraction study of the layered compounds $\mathrm{MnPSe}_{3}$ and $\mathrm{FePSe}_{3}$, Solid State Commun. 40(12), 1067 (1981)

37. T. Zhu, J. Li, Ultra-strength materials, Prog. Mater. Sci. 55(7), 710 (2011)

38. A. R. Wilds, B. Roessli, B. Lebech, K.W. Codfrey, J. Phys.: Condens. Mater 10(28), 6417 (1998)

39. K. Okuda, K. Kurosawa, S. Saito, M. Honda, Z. Yu, M. Date, Magnetic properties of layered compound $\mathrm{MnPS}_{3}$, J. Phys. Soc. Jpn. 55(12), 4456 (1986)

40. N. Sivadas, M. W. Daniels, R. H. Swendsen, S. Okamoto, D. Xiao, Magnetic ground state of semiconducting transition-metal trichalcogenide monolayers, Phys. Rev. B 91(23), 235425 (2015)

41. J. B. Goodenough, Theory of the role of covalence in the perovskite-type manganites [La, M(II)] $\mathrm{MnO}_{3}$, Phys. Rev. 100(2), 564 (1955)

42. J. Kanamori, Crystal distortion in magnetic compounds, J. Appl. Phys. 31(5), S14 (1960)

43. M. A. Subramanian, A. P. Ramirez, W. J. Marshall, Structural tuning of ferromagnetism in a 3D cuprate perovskite, Phys. Rev. lett. 82(7), 1558 (1999)

44. W. B. Zhang, Q. Qu, P. Zhu, C. H. Lam, Robust intrinsic ferromagnetism and half semiconductivity in stable two-dimensional single-layer chromium trihalides, J. Mater. Chem C 3(48), $12457(2015)$

45. M. Joe, H. Lee, M. M. Alyörük, J. Lee, S. Y. Kim, C. Lee, J. H. Lee, A comprehensive study of piezomagnetic response in $\mathrm{CrPS}_{4}$ monolayer: mechanical, electronic properties and magnetic ordering under strains, Journal of Physics: Condens. Matter 29(40), 405801 (2017) 


\section{Figure captions}

Fig. 1. (a) Top and side views of monolayer $\mathrm{MnPSe}_{3}$. The unit cell is indicated by red solid line. The grey, purple and green spheres represent the $\mathrm{P}, \mathrm{Mn}$, and Se atoms, respectively. (b) Atomic structures of a distorted $\mathrm{MnSe}_{6}$ octahedron and $\left[\mathrm{P}_{2} \mathrm{Se}_{6}\right]^{4-}$ bipyramids enclosed with hexagonal Mn atoms. Isosurface plots of the spin charge density for (c) FM and (d) AFM configurations. The isosurface value is set up to $\pm 0.1 \mathrm{e} / \AA^{3}$. The blue and red isosurfaces represent spin-up and spin-down charge densities, respectively. (e) Four possible spin configurations and their relative energy $\Delta E$ (meV/unitcell) with respect to the Néel-AFM configuration.

Fig. 2. (a) Variation of the energy difference between the AFM and FM coupling and the nearest Mn-Mn distance in biaxial strained systems. Negative value indicates that the FM configuration is less stable than the AFM configuration. (b) Variation of the band gap at different biaxial strains. (c) Schematic plot of the effect of biaxial strain on the magnetic coupling.

Fig. 3. Band structures and density of states for monolayer $\mathrm{MnPSe}_{3}$ at the biaxial strain of (a) $-5 \%$, (b) $0 \%$, (c) $5 \%$, (d) $10 \%$, (e) $13 \%$ and (f) $15 \%$. Fermi level is indicated by the vertical solid line and set to zero. 
Fig 4. Schematic diagram of possible paths for the (a) NN, (b) second NN and (c) third NN magnetic exchange interactions in monolayer $\mathrm{MnPSe}_{3}$. 
Fig. 1, Q. Pei et al.

(a)

0

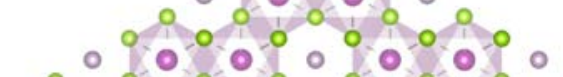

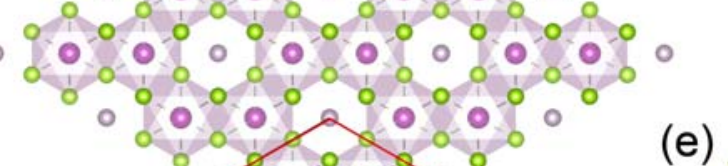

$\sim_{0}^{a_{k}}$

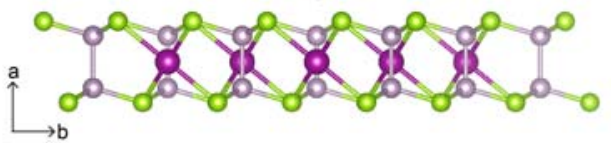

$\circ \mathrm{P} \circ \mathrm{Mn} \circ \mathrm{Se}$

(b)
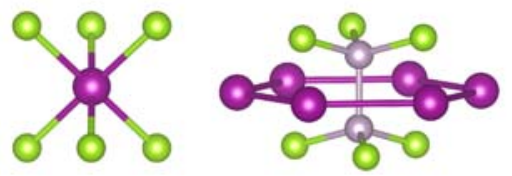

$\mathrm{MnSe}_{6}$ (c)

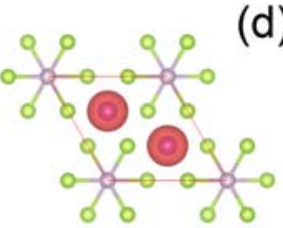

(d)

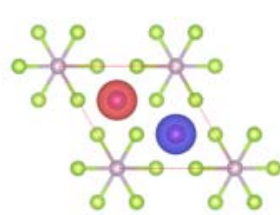

(e)

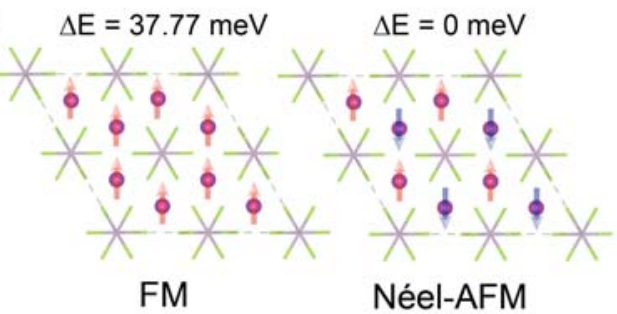

$\Delta \mathrm{E}=13.12 \mathrm{meV} \quad \Delta \mathrm{E}=17.92 \mathrm{meV}$

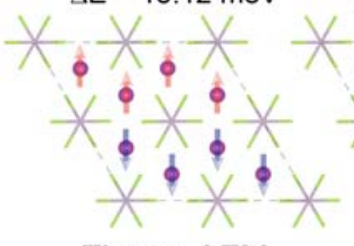

Zigzag-AFM

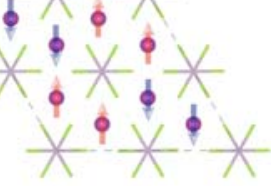

Stripy-AFM 
Fig. 2, Q. Pei et al.

(a)

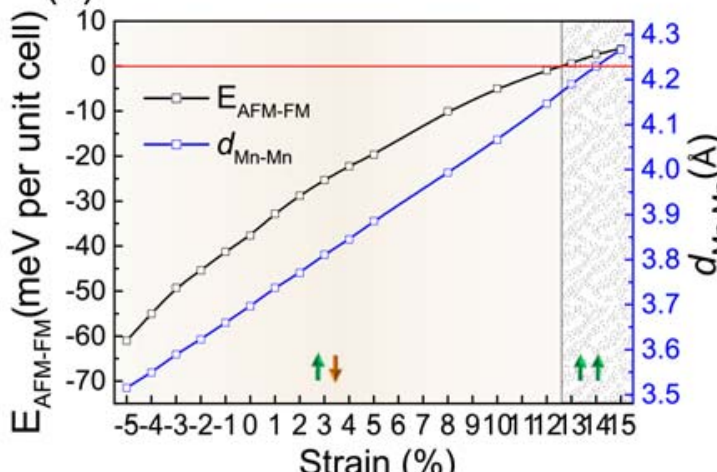

Strain (\%)

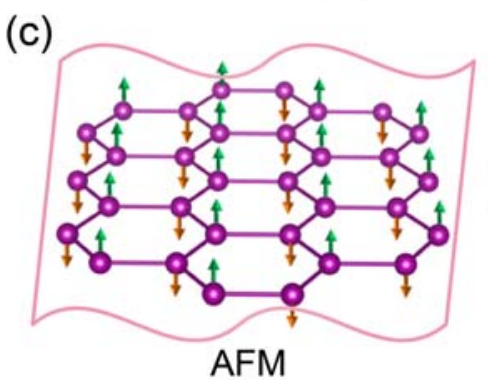

(b)

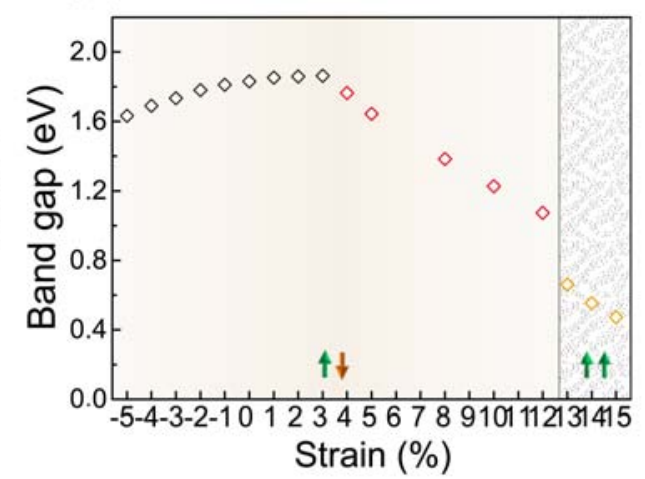

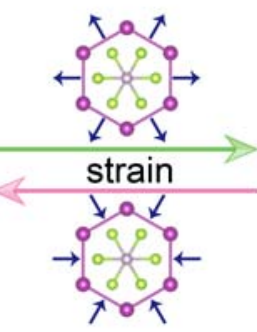

tro

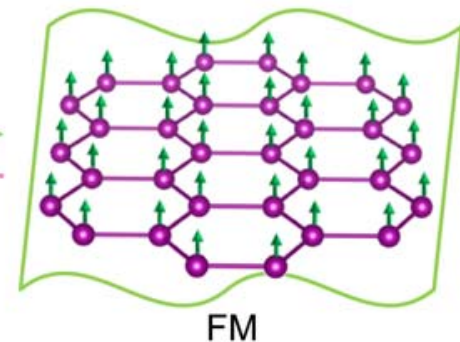


Fig. 3, Q. Pei et al.

(a)

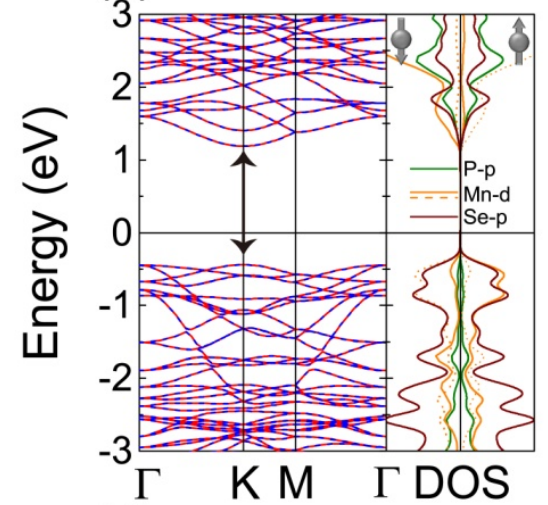

(d)

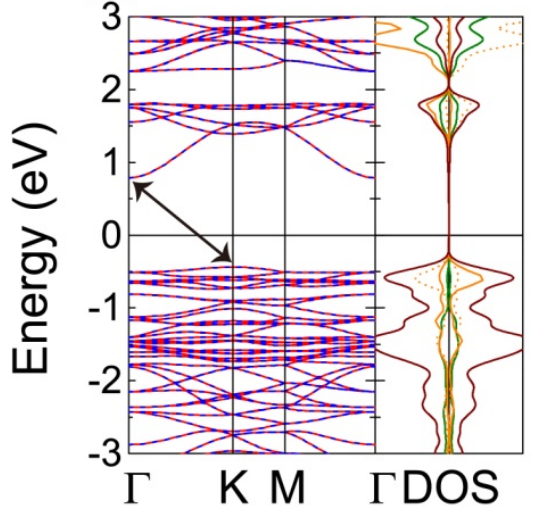

(b)

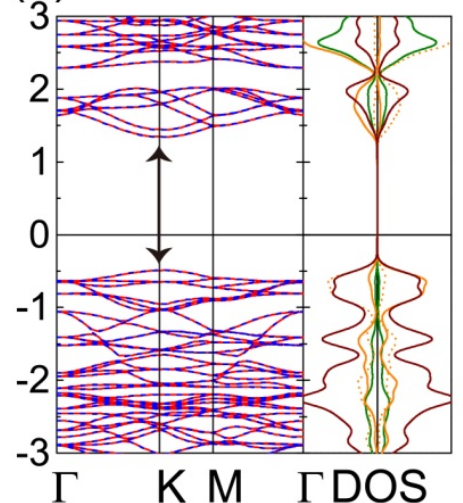

(e)

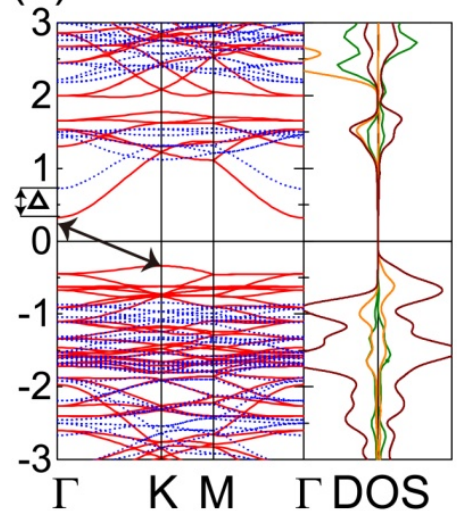

(c)

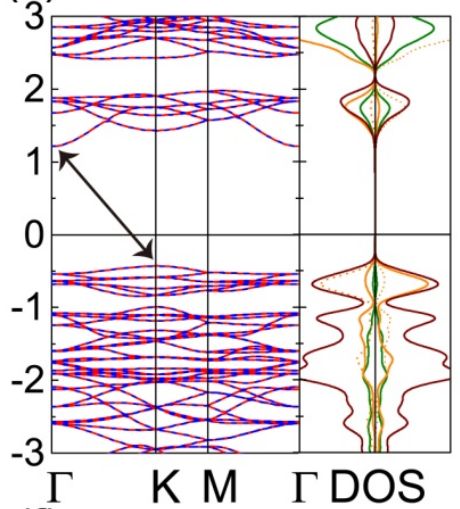

(f)

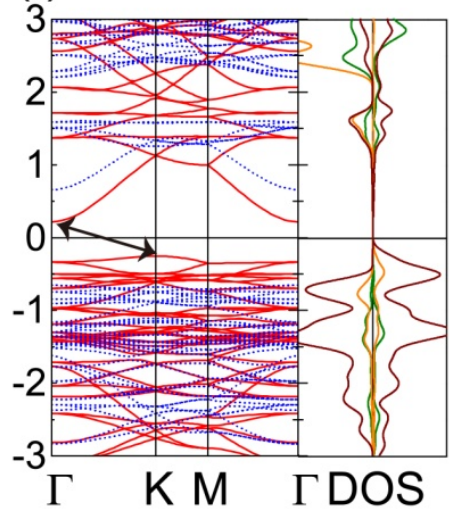


Fig. 4, Q. Pei et al.

(a)
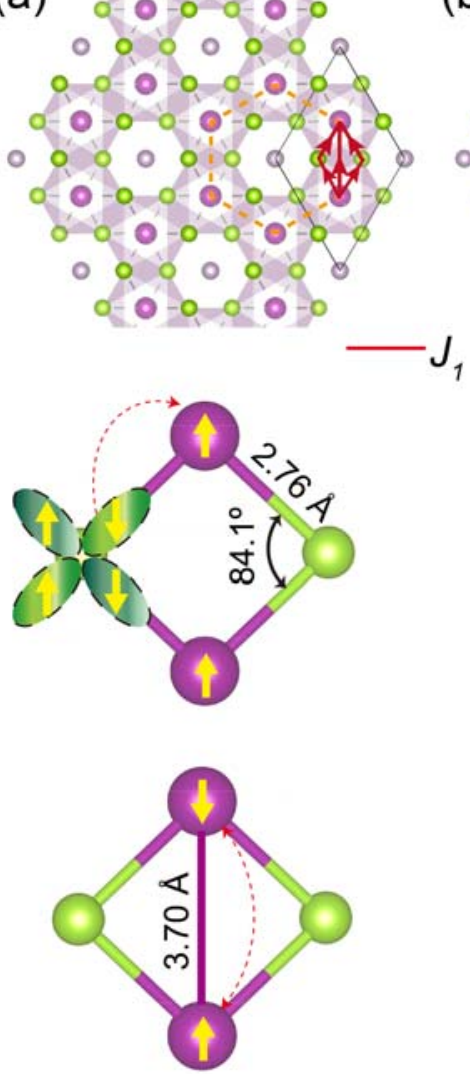

$J_{1}: A F M$ (b) $\circ 0000$
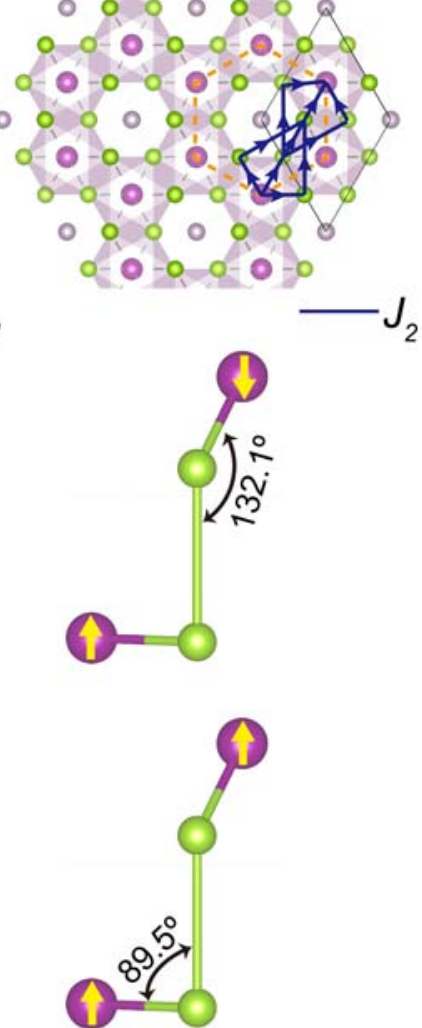

$J_{2}: A F M$ (c) 0000
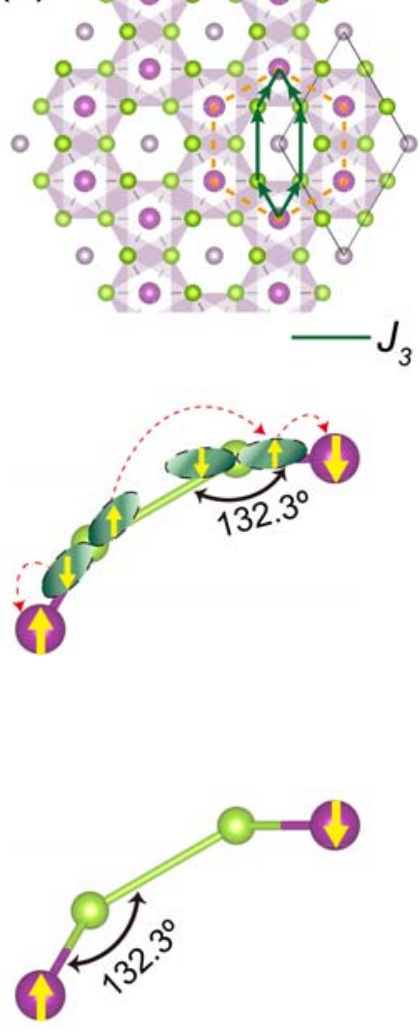

$J_{3}: A F M$ 


\title{
Supplementary Information
}

\section{Tunable Electronic Structure and Magnetic Coupling in Strained Two-Dimensional Semiconductor $\mathrm{MnPSe}_{3}$}

\author{
Qi Pei ${ }^{1}$, Xiaocha Wang ${ }^{2}$, Jijun Zou ${ }^{3}$, Wenbo $\mathrm{Mi}^{1,{ }^{1}}$ \\ ${ }^{1}$ Tianjin Key Laboratory of Low Dimensional Materials Physics and Preparation Technology, \\ School of Science, Tianjin University, Tianjin 300354, China
}

${ }^{2}$ School of Electrical and Electronic Engineering, Tianjin University of Technology, Tianjin 300384, China

${ }^{3}$ Key Laboratory for Green Chemical Technology of the Ministry of Education, School of Chemical Engineering and Technology, Tianjin University, Tianjin 300354, China

\footnotetext{
*Author to whom all correspondence should be addressed.

E-mail: miwenbo@tju.edu.cn
} 


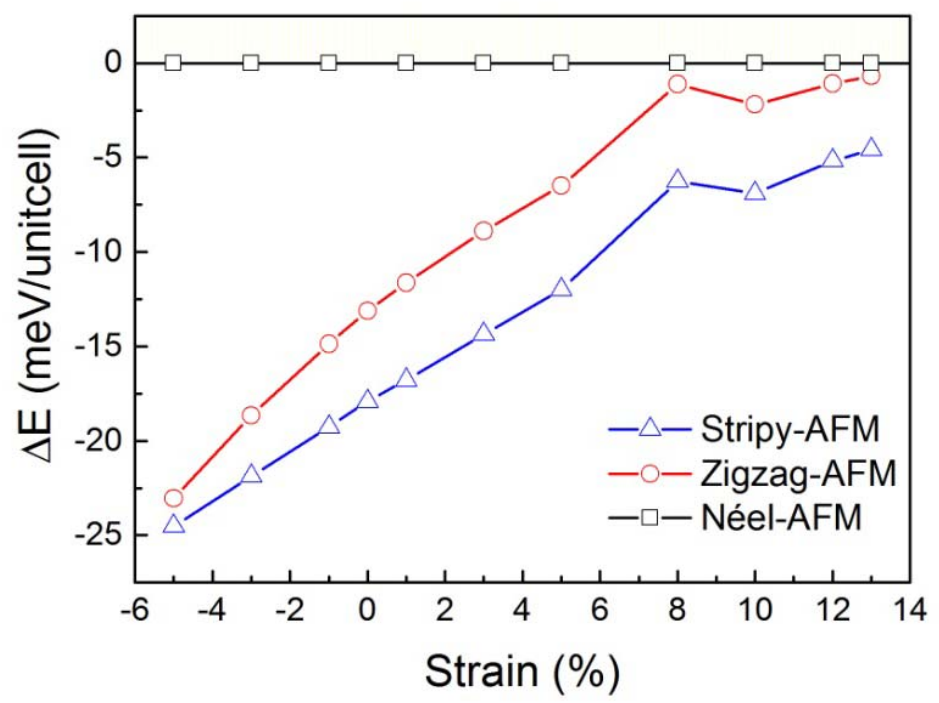

Fig. S1 Variation of total energy relative to the Néel-AFM configuration among Néel-AFM, zigzag-AFM, stripy-AFM. Negative value indicates that zigzag-AFM and stripy-AFM configurations are less stable than the Néel-AFM configuration.

Fig. S1 shows the variation of the energy difference between the regular AFM (Néel-AFM) and other AFM configurations (stripy-AFM and zigzag-AFM) for biaxial strained monolayer $\mathrm{MnPSe}_{3}$. Negative values indicate that other AFM configurations are less stable than the regular AFM configuration. Within the biaxial strain range from $-5 \%$ to $13 \%$, the values of $\Delta E$ almost increases monotonically with biaxial strain. When the biaxial strain increases to $13 \%, \Delta E_{\text {Néel-zigzag }}$ is relatively smaller with the value of $-0.69 \mathrm{meV}$. However, the spins of $\mathrm{Mn}$ atoms with Néel order always possess the lowest energy in the three AFM arrangements, showing a structural stability. Hence, within the biaxial strain range we considered, there is neither a magnetic phase transition from Néel to other AFM phases nor a transition from other AFM phases to FM phase. 
Table S1 Lattice constant $a(\AA)$, magnetic ground state (GS) and exchange coupling constants (meV) in pristine and biaxial strained monolayer $\mathrm{MnPSe}_{3}$. The $a b$ initio calculations are performed within GGA+U+D2 $(U=5 \mathrm{eV}$ for Mn-3d orbital).

\begin{tabular}{cccccc}
\hline \hline & $a(\AA)$ & GS & $J_{1}(\mathrm{meV})$ & $J_{2}(\mathrm{meV})$ & $J_{3}(\mathrm{meV})$ \\
\hline $\mathrm{MnPSe}_{3}$ & 6.403 & Néel & 0.195 & 0.020 & 0.103 \\
$\mathrm{MnPSe}_{3}(1 \%$ strain) & 6.467 & Néel & 0.159 & 0.014 & 0.095 \\
$\mathrm{MnPSe}_{3}{ }^{[16]}$ & 6.334 & Néel & 0.231 & 0.021 & 0.141 \\
\hline \hline
\end{tabular}

Table S2 Lattice constant $(\AA)$, magnetic ground state (GS) and total energy (meV/unitcell) relative to the Néel-AFM configuration among Néel-AFM, zigzag-AFM, stripy-AFM and FM configurations in uniaxial strained monolayer $\mathrm{MnPSe}_{3}$.

\begin{tabular}{cccccccc}
\hline $\begin{array}{c}\text { Strain } \\
(\%)\end{array}$ & $a(\AA)$ & $b(\AA)$ & GS & $\begin{array}{c}\text { Néel } \\
(\mathrm{meV})\end{array}$ & $\begin{array}{c}\text { Zigzag } \\
(\mathrm{meV})\end{array}$ & $\begin{array}{c}\text { Stripy } \\
(\mathrm{meV})\end{array}$ & $\begin{array}{c}\text { FM } \\
(\mathrm{meV})\end{array}$ \\
\hline$-5 \%$ & 6.083 & 6.403 & Néel & 0 & -23.84 & -20.82 & -49.99 \\
$-3 \%$ & 6.211 & 6.403 & Néel & 0 & -18.98 & -19.86 & -44.30 \\
$-1 \%$ & 6.339 & 6.403 & Néel & 0 & -14.81 & -18.60 & -39.77 \\
$1 \%$ & 6.467 & 6.403 & Néel & 0 & -11.64 & -17.29 & -36.12 \\
$3 \%$ & 6.595 & 6.403 & Néel & 0 & -9.503 & -15.96 & -33.37 \\
$5 \%$ & 6.723 & 6.403 & Néel & 0 & -7.988 & -14.43 & -31.09 \\
$7 \%$ & 6.851 & 6.403 & Néel & 0 & -7.025 & -12.54 & -28.82 \\
$10 \%$ & 7.04 & 6.403 & Zigzag & 0 & $\mathbf{0 . 4 3 3}$ & -7.958 & -24.71 \\
\hline \hline
\end{tabular}

Table S2 lists the lattice constant, GS and total energy relative to the Néel-AFM configuration among Néel-AFM, zigzag-AFM, stripy-AFM and FM configurations in uniaxial strained monolayer $\mathrm{MnPSe}_{3}$. Generally, both the uniform biaxial and uniaxial strains could trigger the modification in 
total energies of different magnetic phases and result in the magnetic phase transition. However, in monolayer $\mathrm{MnPSe}_{3}$, the effect is weaker for uniaxial strain by comparing with the variation of total energies in biaxial strained system. With the uniaxial strain changing from $-5 \%$ to $10 \%$, the energy difference between AFM and FM configurations only changes from 49.99 to $24.71 \mathrm{meV}$. Hence, a relatively large uniaxial strain is required to alter the AFM-FM transition. Additionally, we notice a clear transition from Néel-AFM to zigzag-AFM when the uniaxial strain increases to $10 \%$, which can be ascribed to the small energy difference between the two magnetic phases. 